INOUE, T. e ORGEL, L.E. (1983a), The Oligomerization of 2-MeImpG on Poly(C)-An RNA Polymerase Model, J. Mol. Biol., em impressão.

INOUE, T. e ORGEL, L.E. (1983b), A Non-Enzymatic RNA Polymerase Model. Science, 219: 859-862.

LohrmanN, R. e Orgel, L.E., 1973. Prebiotic Activation Processes. Nature, 244: 418-420.

Miller, S.L. e Orgel, L.E. (1974), The Origins of Life on the Earth (Prentice-Hall, New Jersey) 229 pp.

NodA, H., Editor; 1978. Origin of Life. Center for Academic Publications Japan, 637 pp.

Oró, J., Miller, S.L., Ponnamperuma, C. e Young, R.S., Editores; 1974. Cosmochemical Evolution \& the Origins of Life. Volumes I e II. D. Reidel, Dordrecht, 523 and 334 pp.

SCHuster, P. (1981), Prebiotic Evolution. In Gutfreund, H. (ed.), Biochemical Evolution, Cambridge University Press, pp. 15-87.

SshwarTZ, A.W. e GoverDE, M., 1982. Acceleration of HCN Oligo- merization by Formaldehyde and Related Compounds: Implications for Prebiotic Syntheses. J. Mol. Evol., 18: 351-353.

SchwartZ, A.W., 1974. An Evolutionary Model for Prebiotic Phosphorylation.

In: K. Dose, S.W. Fox, C.A. Deborin e T.E. Pavlovskaya (Editores), The Origin of Life and Evolutionary'Biochemistry. Plenum, New York, pp. 435-443.

SchwarTZ, A.W., 1983. Chemical Evolution: The First Stages. Naturwissenschaften, em publicação.

Schwartz, A.W., Joiosten, H. e Voet, A.B., 1982. Probiotic Adenine Synthesis via $\mathrm{HCN}$ Oligomerization in Ice. BioSystems, 15: 191-193.

VOET, A.B. e SCHWARTZ, A.W., 1981. HCN Oligomerization-Isolation and Preliminary Characterization of A New Precursor of Adenine.

In: WOLMAN, 1981, pp. 217-223.

Wolman, Y., Editor; 1981. Origin of Life. D. Reidel, Dordrechet, 613 pp.

\section{LABORATÓRIOS DE QUÍMICA INSTALAÇÃO E FUNCIONAMENTO}

Iniciamos hoje a publicação duma série de artigos sobre a instalação e funcionamento de laboratorios de química. $O$ primeiro artigo desta série é um fac semile dum trabalho da autoria de Ferreira da silva, publicado no Correio Médico em 1893, sobre o Laboratório Municipal de Química do Porto.

Por 1881 o Professor A.J. Ferreira da Silva, a quem está ligada a fundação da Revista e da Sociedade Portuguesa de Química, terminou a análise das águas do rio Sousa que a Câmara Municipal do Porto pretendia então utilizar para abastecimento da cidade. O sucesso alcançado com este projecto levou a mesma Câmara Minicipal a reconhecer a necessidade de laboratórios para controlo químico da qualidade e a criar no ano seguinte, 1882 , o Laboratório Municipal de Química do Porto, para a direcção do qual nomeou aquele ilustre químico. Os laboratórios foram então modelarmente equipados para a época e neles foram realizados numerosos e importantes trabalhos no campo da Química, nomeadamente sobre química legal e toxicologia e sobre química aplicada à higiene, à alimentação, à hidrologia, à farmácia, à indústria, ao comércio e à agricultura.

Pouco tempo após a sua fundação, o Laboratório Municipal de Química do Porto veio também a ser uma verdadeira escola de pós-graduação em Química, concedendo estágios de dois anos a licenciados, especialmente em medicina e farmácia. Por razőes aparentemente pueris, este laboratório veio a ser extinto vinte e cinco anos após a sua fundação, ainda com Ferreira da Silva a ocu-
Hernâni Maia

Universidade do Minho par o lugar de director, deixando assim um vazio que nunca foi preenchido. O seu espólio transitou para a Faculdade de Ciências do Porto, aonde, pelo menos até aos anos sessenta, foi encontrada utilidade para uma boa parte dos respectivos equipamentos.

Entre 1884 e 1885 Ferreira da Silva publicou diversos artigos nas revistas Saúde Pública e La Nature, descrevendo o Laboratório Minucipal de Química do Porto e o seu funcionamento. Porém, após uma ampliação das instalaçōes com vista ao controlo da qualidade do gás de iluminação da cidade do Porto, instalado em 1889, o ilustre químico portuense veio a publicar novo artigo no Correio Médico, descrevendo então a nova forma das instalações e o seu funcionamento.

Em justa homenagem a Ferreira da Silva e em comemoração, se não do primeiro século da exemplar criação do Laboratório Municipal de Química do Porto, que se completou no ano passado, mas pelo menos dos trabalhos da sua instalação, e também em comemoração dos noventa anos da publicação do artigo atrás referido (1893-1983), aqui o publicamos em fac semile.

Estando o nosso país a atravessar, desde há alguns anos, um período de construção e instalação de novos laboratórios de química e prevendo-se o interesse que poderá vir a ter a divulgação daqueles já instalados, com este trabalho de Ferreira da Silva iniciamos uma série de artigos neste domínio. Assim, no próximo número do Boletim publicaremos um trabalho sobre o Laboratório de Química da Universidade do Minho, e aqui deixamos desde já um convite para que nos sejam enviados novos trabalhos para publicação nesta série. 


\section{Publication du Corroio Medico do Lisboa}

\section{LE LABORATOIRE MUNICIPAL \\ $D E$}

\section{CHIMIE DE PORTO}

Note nédite de Mr. le i'Rofessetr A. J. Ferkeira da Silva

Traduction française, rerue par 1'auteur (Correio Medico, 1893, u. " 4, p. 26-30)

L ISBOA

Typographia de Christovão Augiesto Robrigletes

Go, Rua de S. Paulo, G2

$1 \overline{893}$ 


\section{Le Laboratoire Municipal}

DE

\section{CHIMIE DE PORTO}

Traduction d'une note inédite, revue par l'auteur

(Correio Medico de Lisboa, 1893, n.* 4, p. 26-30)

Le Lahoratoire Municipal de Porto, dont la Saude Publica ${ }^{1}$ et La Nature ${ }^{2}$ ont déja donné une description, a éprouvé les années dernières des modifications ímportantes et a été agrandi de nouveaux aménagements, ce qui nous fait tenir à en donner une nouvelle description, d'après son état présent.

I. - Tout le batiment siège sur les terrains au nord de l'Hôtel de Ville. Un petit jardin y donne accès, aussi bien qu'au laboratoire de bactériolngie, auquel il est adossé. De ce jardin, représenté au bas de la Fig. 1, on pénètre dans le Laboratoire par un petit vestibule et un corridor, sur les côtés desquels on voit:

A droite: a) un cabinet, avec guichet sur le vestibule (1), dans lequel le public dépose les produits à faire analyser; on y voit aussi le vestiaire du personnel de l'établissement; $b$ ) une salle-dépòt (3) pour les types des denrées alimentaires et des substances employées à leur sophistication; on y a accommodé deux lavabos et le bureau du Secrétaire; $c$ ) une autre salle (5), le maqaasin de la verrerie - surtout des verres destinés aux essais volumétriques: pipettes, burettes, ballons jauqés, éprouvettes graduées, etc. - et des ustensiles de porcelaine et de grès.

A gauche: a) le cabinet du Directeur (2), contenant la bibliothèque, une table pour les microscopes, etc; $b$ ) une salle (4), servant à emmagasiner les produits chimiques. Au milieu de cette salle, une tahle à armoires bien fermées reçoit les réactifs pouvant s'altérer par la lumière. Une armoire spéciale contient les liqueurs titrées pour analyses volumétriques, et d'autres ar-

' Saude Pullica, 1.• anno, 188i, p 219, 237, 266, 299, 310 e 326.

'La Nature, 11.' année, 1885-86, N.o 654, du 12 Décembre, 188j, p. 22 et 23. 


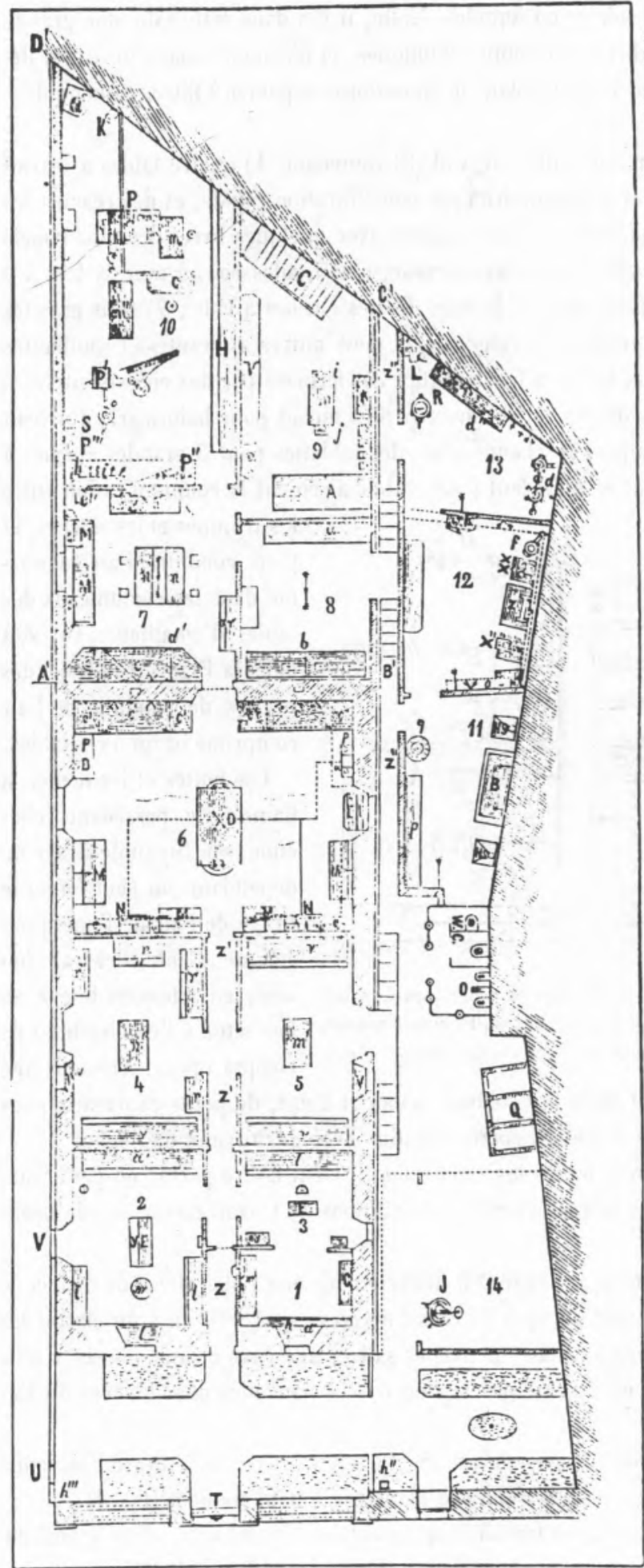

* 1, Piser pour ríception den ielsantillons analyser: $G$, vektialre; $a$, armoire; $h$, com. pteur is gaz; $m$, talile.

2 Cabinet du lirecteur; $k$, bnresu aver

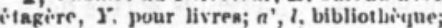
$m$, table.

3, Salle-dépôt ; $K$, burean; $l$, vitrines avee collections de type*s de denréen alimentaires et de nubatances employées ì leur sophistieatiou; $y$. lavabo.

4, Magarin de produits chimiques: $a$, ar moire; $m$, table; $r, r \cdot r, " r,{ }^{\prime}$ armoirek is rizetifx liquides; $r, "$ "' armoire pour produit organiques; $v$, armoire a reactifk nolides.

5 , Maganin de la verrerie; $m$,' table; $r$. nrmoires a verrerie et à porcelaine, $r$ te: $v$ étagères à verrerie'; $v$, armoire it iustrument et ì verrerie graduin.

6 , Grande salle à travail; $D$, inble pour distillations et pour les grandes cagen a ex sicention; $\boldsymbol{E}$, burrau; $\boldsymbol{M U}$, tablex a travail $x$, rables d cmailleur, 1, Tetonk pour l'air

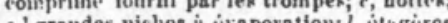
Cor livere; po pur tiv rea po. I.Ussac à eau; $\alpha, \beta$, trompes asplrantes et aspirantes-foulantes d'ALNERGKIAT.

7 , Seconde salle it travail; $M$, tables it travail; $a$, armoire à réactifs; $c$, botte; $d$ ', soxval; g, table à esaai de la purcté du gaz d'éclairage; $n$, bureau il quatre places; $r$, vitrine á produits chimiques.

8 , Salle des balances; $b$, table aux balauces; $u$, armoires à ustensiles.

9 , Salle à analyser les gaz; $A$, grande vi trine at appareils; $C$, escalier descendan dans ia cave; $K$, table pour instruments mesurer le gaz; $V$, annoires à verrerie; $i$, cuve in mercure; $m$, table.

10 , Grande chambre noire; $I I$, table et igare a inktruments, etc.; $K$, eloche pour cerifier l'elat d'epuration du gaz d'éclaira.

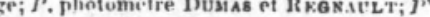
photonitre Desas et REGSALLT modific: "." photomitre Buxs kN; r.'" vírificatenr incuun; $S$. indieateur de preskion du ga celairage; c, burean; i, rampes pour l'eskai den becs in a $\mathrm{az} ; m, \mathrm{tabic}$ pour spectrosenpes et polarimitrex; $y$ lavabo.

II, Cabinet il lavages et it opirations ave acilie kulfhydrique; $B$, grande ange en ardoike pour nettoyagrs, et, au dessous, réker. voir à eau pour lè crémomìtres; $H S$, en. ceintes vitries (Digestoria) pour les appareils it hydregine sulfurí; $p$, étagieres: égouttuir-bérisson à colonue galvaniséc.

12, Chambre à foumeanx et a analyses organiques; $\boldsymbol{F}$, four Courtoxxk, à incinéra. tiou; $f$, fourneau ì vellt; $x$, four à mouffles. our incinirations, avec bruleur à gaz; $x$, fourneau PвRrot; $x$ " grand fourneau Dvpus, à incinérations; $z$, table pour analyses organiques.

13, Chambre à filtration et distillatiou de Teau; $L$, tiltre Chaунzulasd en faience, trois bougies en porcelaine, avee réscrvoir pour Teau fitrie, $k$, réservoir en fer. il papols maillces. pour pau filtrie, d, Lrand alambic, chaufie au gaz, pour distillation de Yeau; d, ablettrs pour petits alambies.

14, Cour pour opérations à l'air libre; $J$, graud gazomintre de 360 litres; $O$, urinoirs; $Q$, cages a lapins; $W C$, water; closets.

$Z$, petit vestibule; $Z \cdot Z$, corridor; $Z$ ', passagex a l'air libre; $B$.' escalier; $V$, porte de communication avec le laboratoire de baetériologie; $T$, entréc du jardin; $U$, entríe du laboratoire de bactíriologie; $h, " h$," compteurs it ean.

rig. 1 - Plan du Laboratuire (Échelle de ${ }^{1}{ }_{200}$ ) * 
moires sont destincés aux réactifs solides ou liquides. Enfin, il y a dans eftte salle une grande boite i réactifs surmonté d'une vitrine i produits chiminues, et d'armoires où se trouvent dé. posies les diffirentes sortes de papiers usités dans le laboratoire - papier à liltre, papier à dỵaliser, papiers réactifs, etc.

Le corridor ahoutit a une grande salle a travail (6) contenant: 1) quatre tables à travail (lig. 9), nunies de robinets a eau et i gaz, de trompes pour filtration rapide, et des réactifs les plus usuels: 2) deux tables d'énailleur; 3) une étagère avee quelyues livres des plus usuels dans le lahoratuire; 4) deux grandes étuves Gar-Lussac, à eau, adossées au mur, a 4 et à 9 portes; 5 ) une grande table pour distillations et lavages; 6 ) des cloches a vide ; 7) trois grandes trompes i eau, dont une double, systeme Alvengxiat, et denx autres aspirantes ot souflantes d'Alvengniat el Muencike; 8) deux hottes a lenètres (fiz. 3), fermées par des châssis vitrés, à contre poids : 9) a cote de chacune de res hottes, deux petites niches pour bain-marie a niveau constant; 10) au dessus des trumpes, de chaque côti, des tablettes pour 3 grandes cloches à évaporation dans le vide; 11) tout ce qu iil faut pour établir aisément la communiration entre

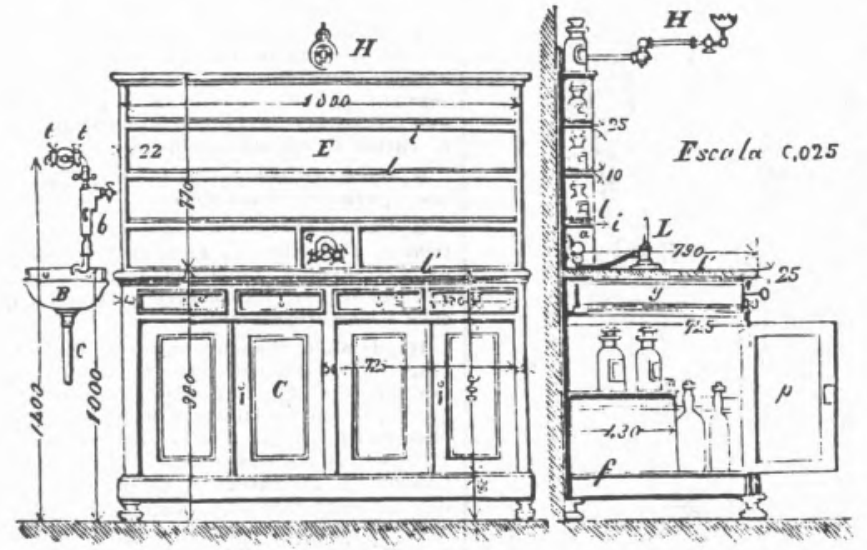

Fig. 2 - Table a trarail (èlevation, vue de face et coupe transversale). $H$, Becs à gaz; $E$, rayons des étagères à réactifs; $l . l, l$, Plaques d'ardoise;

$b$, Trompe Alvkginat; $\boldsymbol{B}$. Bassin d'écoulement des eaux; $t$, robinet à eau. les trompes et les cloches, et pour conduire l'air comprimé dans les chalumeaux des tables d'émailleur. On voit dans la Figure 1 le trajet des tuyaux de conduite de l'air comprimé jusqu'a ces tables.

Les hottes et les niches à hain-marie possédant, chacune, son tuyau de tirage in. dépendant, on peut forcer le tirage de l'air de l'une quelconque d'entr'elles, au besoin, en allumant le gaz an bec situé à l'embouchure de chaque tuyau. Ajoutons que ces hottes et ces niches présentent aussi des robinets i eau et à gaz, de petits caniveaux pour l'écoulement des eaux sales, et des grilles en communication avec des tuyaux de l'égout.

L'ancien Laboratoire comprenait toutes les pièces dont il vient d'ètre parlé; on peut voir, cependant, qu'elles ont été désencombrées et que les conditions de travail en ont été de beaucoup améliorées.

II. - La Municipalité de Porto ayant chargé le Directeur de son Laboratoire de diriger le servi:e de vérilication du gaz d’éclairage, qu elle venait de créer en 1889, on a dú élargir les locaux destimés aux travaux ehimiques; quatre nouvelles salles, une cave et une annexe y ont, donc, été ajoutées. Cè ıui suit est une courte description de ces nouvelles dépendances du Laboratoire.

Les quatre nouvelles salles, dont le sous-sol est occupé par la cave, font suite i l'ancienne salle à travail, raṇ̨ées comme on peut le voir dans la plante de l'établissement (fig. 1).

A) La première de ces salles, salle à travail supplémentaire (7), en outre d'un grand bureau disposé pour l'écriture et la lecture, contient deux tables à travail, une hotte, des étuves, 
un alambic-étuve de Moitessisn et une table sur laqueile sont disposés les appareils pour l'issai chimique du gaz d’éclairage.

B) La deuxième, c'est la salle aux balances (8). Elle se trouve à droite de la prenicire et contient, en outre des balances, une armoire pour les exsiccateurs ct des tablettes, destinćes à quelques ustensiles.

C) La troisiime (10) est une grande chambre noire, à parois peintes en noir mat, donnant aisément une obscurité complète. On y a installé le photomètre, très connu, de Dumas et RE.

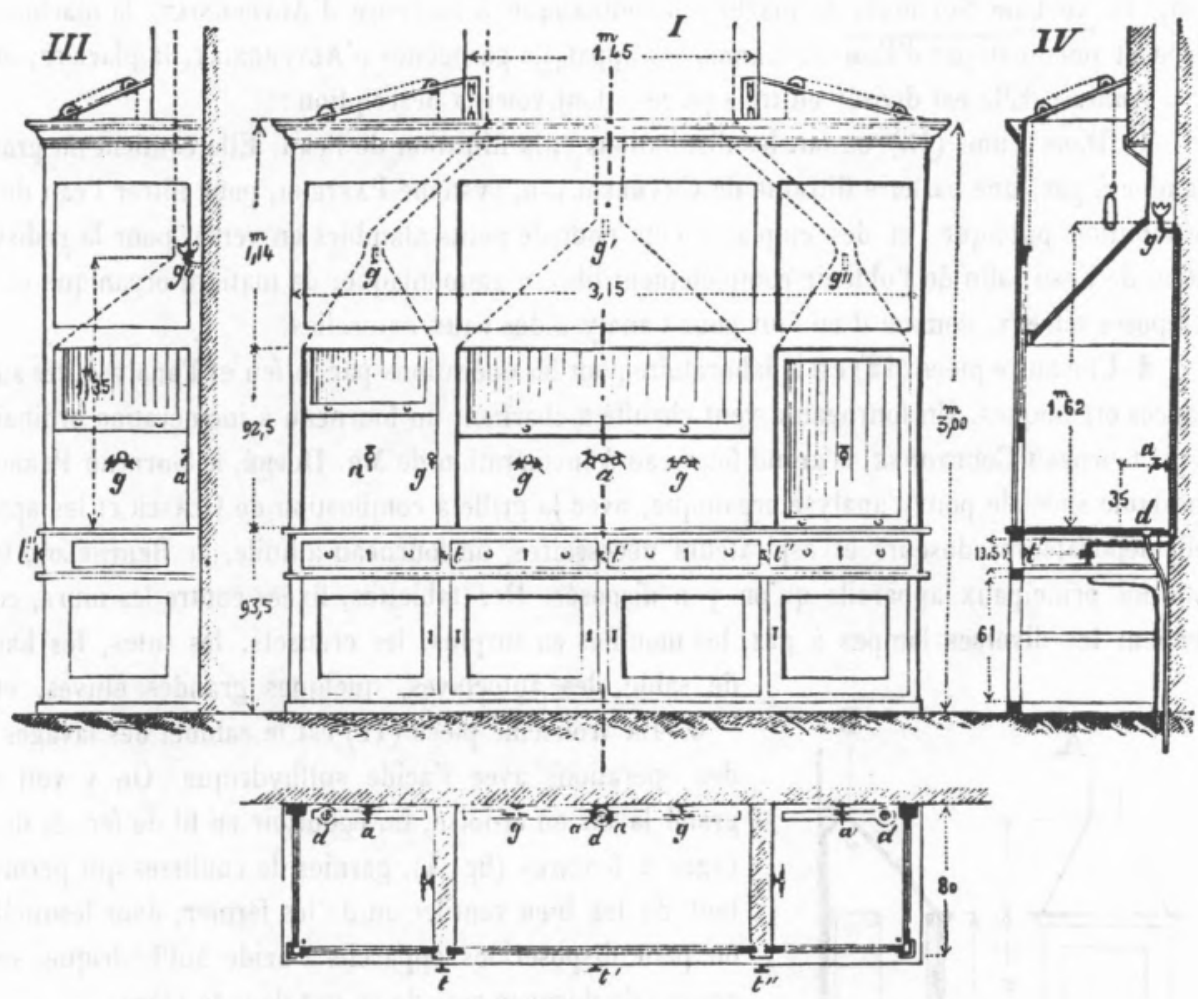

rig. 3-Hotte el viches (plan, èlévation en face et à côté. coupe). a, Robinet ì eau; $g$, Rohinets à gaz; t, t,' t," Robinets à longue clef, servant à ourrir ou á fermer le gaz qui alimente les becs de tirage g. g.' $g " ; d$, Egrilloir en communication avec les tuyaux d'égout et avec les rigoles creusées dans l'ardoise.

gxault, modèle usité à Paris, aussi bien que dans un grand nombre de villes, pour la définition du pouvoir éclairant du gaz d'éclairage. Un autre modèle, le photométre de llusas et REGsavet, modifi', s'y trouve anssi, il còte du photomètre de Bussex et d'un vérificateur de Ginot: donnant la valeur approchée du pouvoir éclairant du gaz; enfin, il y a un indicateur-registreur de la pression du gaz et une rampe pour l'essai des bees employés dans l'éclairage.

Cette chambre nuire sert, au besoin, aux essais d'analyse spectrale ou polarimétrique, et, à cet effet, elle possède une table munie de robirets à gaz, sur laquelle les spectroscopes et les saccharimètres peuvent étre installés très commodément. Contre le mur de droite il y a une longue table et des tablettes, recevant plusieurs appareils du Laboratoire. 
D) La quatrième des nouvelles salles, en communiration direcie avec la seconde salle á travail, est spécialement destinée aux analyses des gaz (9). En face de la fenétre qui regarde le nord on y voit, sur une table, l'eudiomètre de Bunsen. (On y voit aussi une haute cuve it eau, pour les essais avec l'uréomètre d'Yvon, des armoires à verrerie, des vitrines pour appa. reils, etc.

Care. De la dernière salle on descend dans la cave, une large piice, qui est le dépôt de la collection des vins types, des scellés et des échantillons de produits aualỵsés. Les gros appareils y trouvent aussi leur place; et on y voit le laminoir, la presse SAMAIs, la pressu a perius. sion, la turbine Soundat, la machine pmeumatique a mercure d'Alvengsiat, la machine à glace et pneunatique d'Edono Canné, les appareils gazogènes d'Alvengniat, la glacière, ete.

Annexe. Elle est divisée en trois pièces, dont voici la deseription :

1. Jans l'une (1:3) on fait les distillations et la filtration de l'eau. Elle contient un grand alambic a gaz, une batterie filtrante de Chamberland, système Pasteun, pour filtrer l'eau de la distribution publique, et des emplacements pour de petits alambies en verre, pour la redistiltation de l'eau, afin de l'obtenir complétement libre d'ammoniaque, de matière organique et de composés nitreux, comme il en faut pour l'analyse des eaux naturelles.

2. Une autre pièce (12) est le laboratoire pour les opérations par le feu et l'analyse des substances organiques. Un fourneau à vent chauffé à charbon, un fourneau à incinération ordinaire et un fourneau Courtonne, le grand fourneau à incinération de Mr. Dupré, le fourneau Perrot, une table spéciale pour l'analyse organique, avec la grille à combustion de Glaser et les appareils dépurateurs, doseurs el aspirateurs nécessaires, un fourneau a huile, de Benthelot, tels sont les principaux appareils qu'on y a disposés. Des tabletles, fixées contre les murs, contiennent les diverses lampes à gaz, les mouffles en surplus, les creusets, les tutes, les bains

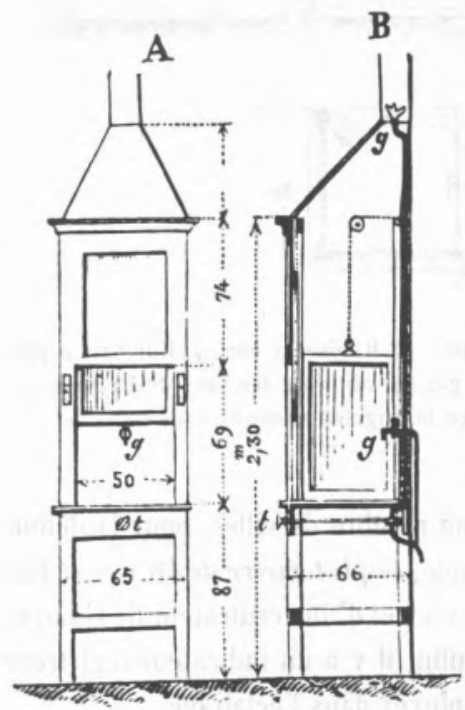

rig. 4-Enceinte vitrée pour $\boldsymbol{H}^{2} \mathrm{~S}$. (èlevation en face el coupe). $g$. Robinet à gaz; $g^{*}$. Bec à tirage: $t$, Robinet à longue clef servant à ourrir ou à fermer le gaz qui alimente le petit bec de tirage $g^{\circ}$. de sable, les autoclaves, quelques grandes étuves, etc.

3. La troisiême pièce (11) est le cabinet des lavages et des opérations avec l'acide sulfhydrique. On y voit un grand lavoir en ardoise, un égouttoir en fil de fer, et deux cages à fenétres (fig. 4), garnies de coulisses qui permettent de les bien ventiler ou de les fermer, dans lesquelles on peut disposer les appareils i acide sulfhydrique, sans crainte de dégagements de ce gaz dans la pièce.

Cour. A côté du laboratoire on trouve, enfin, une cour ouverte (14), destinée aux travaux à l'air libre. En ontre de quelques cages pour les lapins à expériences, elle contient un grand gazomètre, d'une capacité de 360 litres; il sera bientôt employé comme réservoir à gaz d'éclairage, le fournissant, à la pression désirable et par une canalisation spéciale, aux fourneaux PErnot, à incinération, etc., thaque fois que la pression dans la canalisation gévérale de la ville ne sera pas suffisante.

Les différentes salles de l'étalılissement sont fournies de l'eau à l'aide d'un tube général, placé à l'exterieur, le long du mur du batiment, chaque pièce la recevant par un conduit spécial, muni de robinets, ce qui permet de rendre la 
consommation de l'eau dans une queleonyue des piriess tout-it-fait independante de celle faite daus les autres piicres.

Le Laboratoire peut étre alimenté d'eau, soit par une sourcé munieipale, qui la débite sous une faible pression, soit par le réseau publique, qui la fournit sous une pression de plus de 40 mètres. C'est la premiere qui est ordinairement employée pour le nettoyage de la verrerie, etr. lin systime de robinets permet de faire circuler dans les conduites du Laboratoire l'une ou l'autre de ces eaux.

Les tubes conducteurs du gaz et de l'eau, aussi hien que les tuyaux d'égout, sont disposés dans des rigoles cimenties, recouvertes de playues en fer, en sorte qu'ils prurent étre visités. Les diflerentes pièces sont ventilées par des ventilateurs système Lewıshas.

III. - Le personnel privatif du Laboratoire (nous ne mentionnons pas celui qui s'oceupe spécialement de la vérification du gaz) comprend maintenant: 1 directeur; $\boldsymbol{Z}$ aides-chimistes; $\mathbf{z}$ chimistes praticants; et 1 homme de service, auquel un autre a été adjoint. Les appointements en sont bien modestes, conme on preut, du reste, le voir, dans le lodget pour l'annce courante (1893), que voici :

\section{I.-Personnel:}

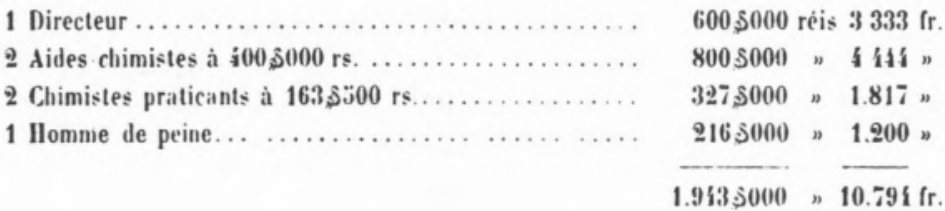

II. - Matéricl - comprenant frais d'expériences, achat d'instruments, de produits chimiques et de serrerie; eau; impression du catalogue du materiel et des livres du laboratoire............. 1.500\$000 rèis $8.333 \mathrm{fr}$

III.-Entretien de locaux, meubles, etc

$1.000 \$ 000$ s.5\%\%”

En tout: 4.433\$000 $n 24.682 \mathrm{fr}$.

IV. - En ce qui regarde la réglementation des services, nous n'aurions que peu de modifications a faire à ce que nous avons exposé dans notre notice de 1884 dans la Saude Publica, et nous dirons seulement quelques mots sur la classe des chimistes praticants, créée en 1889.

Les chınistes praticants sont nommés pour deux ans, après concours. Les candidats qui demandent à ètre inserits au concours sont tenus de présenter: $1 .^{\circ}$, acte de naissance; $2 .^{0}$, certificat de bonne vie et meurs; $3 .^{\circ}$, certificat d'approbation aux examens de chimie ninérale, chimie organique et chimie analytịue, passés devant un établissement d’enseignement supé. rieur. Ils peusent y joindre tout autre document i l'appui de leur aptitude. - Ces candidats passent un examen au Laboratoire Municipal, devant son Directeur, comprenant: $1 .^{\circ}$ Un épreuve orale, consistant en qurstions sur la chimie générale, les falsifications les plus communes des denrées alinentaires, les moyers de les reconnaitre, et les lois et réglements relatifs à la fiscalisation des aliments; $2{ }^{\circ}$ Une épreuve pratique faite au laboratoire. - Sont préférés 
pour ces emplois les èleves en midecine et les élives pharmariens de 1." elasse, pouvant dunner au service du laboratoire, an mons, 3 1/2 heures suivies pendant chayue journer. Apres deux ans complets de service au Laborateire, les chimistes praticants ont droit it un certifieat d'exercice, signé par le Directeur de l'étahlissement.

V. - Soit pour la eréation de ces plares de chimistes praticants, soit pour la riforme du matiriel et du laboratoire le laboratoire est de beaucoup redevable, dans ces dernieres anneres,

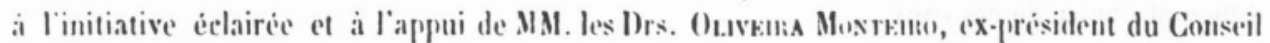
Municipal de Porto, et Sisva Pisto, ex enonseiller Municipal, anxifuels nous tenons a adresser nos remerciments.

Porlo, Janvier 1893.

Le Dnecteun de Labonatume

A. F. Ferrcira da Silua. 